\title{
Confined harmonically interacting spin-polarized fermions in a magnetic field: Thermodynamics
}

\author{
S. Foulon, F. Brosens, and J. T. Devreese* \\ Departement Natuurkunde, Universiteit Antwerpen (UIA), Universiteitsplein 1, B-2610 Antwerpen, Belgium \\ L. F. Lemmens \\ Departement Natuurkunde, Universiteit Antwerpen (RUCA), Groenenborgerlaan 171, B-2020 Antwerpen, Belgium
}

(Received 13 October 1998)

\begin{abstract}
We investigate the combined influence of a magnetic field and a harmonic interparticle interaction on the thermodynamic properties of a finite number of spin-polarized fermions in a confinement potential. This study is an extension using our path integral approach of symmetrized density matrices for identical particles. The thermodynamical properties are calculated for a three-dimensional model of $N$ harmonically interacting spinpolarized fermions in a parabolic potential well in the presence of a magnetic field. The free energy and the internal energy are obtained for a limited number of particles. Deviations from the thermodynamical limit become negligible for about 100 or more particles, but even for a smaller number of fermions present in the well, scaling relations similar to those of the continuum approximation to the density of states are already satisfied. [S1063-651X(99)02604-5]
\end{abstract}

PACS number(s): 05.30.-d, 03.75.Fi, 32.80.Pj

\section{INTRODUCTION}

In the present paper we study the thermodynamical properties of a confined system of spin-polarized fermions in the presence of a magnetic field. The method used is an extension of the combination of the path integral formalism [1] and the method of symmetrized density matrices [2], developed previously [3-6] for a model system of harmonically interacting identical particles (bosons or fermions) in a parabolic well (hereafter for brevity referred to as the harmonic model).

Because of the experimental realization of Bose-Einstein condensation [7-9] and the theoretical work on this phenomenon employing other methods [10-17], full details for this harmonic model with interactions were first worked out for bosons. The model shows the onset of Bose-Einstein condensation in the specific heat [18] for a finite number of particles, and its moment of inertia is drastically reduced below the condensation temperature [19]. An application of the method to real systems can be found in Ref. [20] for ${ }^{87} \mathrm{Rb}$.

The actual calculations for the fermion case require more advanced techniques, such as the generating function approach and the corresponding contour integration, because of a numerical sign problem. In the absence of a magnetic field, explicit results for the thermodynamics and the static correlation functions of the harmonic model of spin-polarized fermions were already obtained with these techniques $[5,6]$.

The harmonic model clearly has intrinsic value on its own, because it is one of the rare examples of an exactly soluble many-particle system with interactions. The physics of the model is relatively straightforward in the sense that it allows for center-of-mass excitations that oscillate at frequencies different from those of the internal degrees of free-

\footnotetext{
*Also at Universiteit Antwerpen (RUCA) and Technische Universiteit Eindhoven, NL 5600 MB Eindhoven, The Netherlands.
}

dom. This property makes it well suited as a trial model for the variational treatment of the thermodynamics of systems with more realistic interactions because the model parameters can be related with the system characteristics with the aid of the Jensen-Feynman inequality [2]. The present paper addresses only the first part of such an approach because it requires also the density and the pair correlation function which we could obtain under the simplifying assumptions of no magnetic field [6]. Furthermore, it provides a testing ground for new approaches to Monte Carlo simulations of interacting fermions such as many body diffusion [21-23]. Especially for quantum dots, it is important to take the magnetic field into account in order to freeze out the opposite spin states. In the present paper we present an extension of the methods mentioned above to harmonically interacting confined fermions in a magnetic field.

The paper is organized as follows. In Sec. II we present the path integral for harmonically interacting particles in a parabolic confinement potential in the presence of a homogeneous magnetic field. This will be done for distinguishable as well as for identical particles. The mathematical details of the calculation for identical particles are given in the Appendix. In Sec. III the permutation symmetry will be taken into account with the aid of the projection technique. The introduction of the permutation symmetry implies the rewriting of the sum over all possible permutations to a cyclic summation [2] which leads to the generating function of the partition function. Specific results for fermions will be presented in Sec. IV. This involves the extraction of the partition function and other thermodynamical quantities from the generating function. Also the ground state energy and the magnetic susceptibility in the zero-temperature limit will be investigated. Additionally we will study the finite number corrections to the thermodynamic limit for the free energy and the internal energy as a function of temperature and magnetic field. In the last section some conclusions are given. 


\section{IDENTICAL OSCILLATORS IN A MAGNETIC FIELD}

The calculation of the path integral for $N$ identical interacting oscillators in a magnetic field is similar to the case without a magnetic field in [3]. This approach crucially relies on the detailed investigation of the classical action and the path integral corrections to this classical action. Alternatively, a more stochastic approach could be followed [24]. The Lagrangian (in atomic units) for $N$ oscillators with harmonic two-body interactions and in the presence of a homogeneous magnetic field is given by

$$
L=\frac{1}{2} \sum_{j=1}^{N}\left(\dot{\mathbf{r}}_{j}^{2}-2 \omega_{c} x_{j} \dot{y}_{j}\right)-V_{1}-V_{2},
$$

where $\omega_{c}$ is the cyclotron frequency and

$$
V_{1}=\frac{\Omega^{2}}{2} \sum_{j=1}^{N} \mathbf{r}_{j}^{2} \quad \text { and } V_{2}= \pm \frac{\omega^{2}}{4} \sum_{j, l=1}^{N}\left(\mathbf{r}_{j}-\mathbf{r}_{l}\right)^{2}
$$

It is obvious that the two-body potential is either attractive or repulsive depending on the plus sign or the minus sign considered in $V_{2}$. The magnetic field introduces a coupling in the plain perpendicular to its direction. This means that we can separate the Lagrangian into two contributions $L=L_{x y}$ $+L_{z}$. The Lagrangian $L_{z}$ simply describes a harmonic oscillator, whereas $L_{x y}$ contains the magnetic field. The Lagrangian can be rewritten in terms of the center-of-mass coordinate $\mathbf{R}(X, Y, Z)$ and the coordinates $\eta_{j}\left(u_{j}, v_{j}, w_{j}\right)$ describing the coordinates of the particles measured from the center of mass,

$$
\mathbf{R}=\frac{1}{N} \sum_{j=1}^{N} \mathbf{r}_{j}, \quad \eta_{j}=\mathbf{r}_{j}-\mathbf{R}
$$

from which

$$
V_{1}+V_{2}=V_{\text {c.m. }}+V, \quad V_{\text {c.m. }}=\frac{1}{2} N \Omega^{2} \mathbf{R}^{2}, \quad V=\frac{w^{2}}{2} \sum_{j=1}^{N} \eta_{j}^{2},
$$

with

$$
w=\sqrt{\Omega^{2} \pm N \omega^{2}} .
$$

For a repulsive two-particle potential the internal frequency $w$ has to satisfy the stability condition that the confining potential has to be sufficiently strong to overcome the repulsion between the particles. We draw attention to the fact that the transformation to the center-of-mass coordinate system diagonalizes neither the Lagrangian nor the Hamiltonian, because of the subsidiary condition

$$
\sum_{j=1}^{N}\left(\mathbf{r}_{j}-\mathbf{R}\right)=0
$$

We obtain the propagator for distinguishable (indicated by a subscript $D$ ) particles from the action expressed in the imaginary time variable $\beta=1 / k T$ and it is written as

$$
\begin{aligned}
& K_{D}\left(\mathbf{r}_{1}^{\prime \prime}, \ldots, \mathbf{r}_{N}^{\prime \prime}, \beta \mid \mathbf{r}_{1}^{\prime}, \ldots, \mathbf{r}_{N}^{\prime}, 0\right) \\
& \quad=K_{D}\left(\left(\bar{x}^{\prime \prime}, \bar{y}^{\prime \prime}\right), \beta \mid\left(\bar{x}^{\prime}, \bar{y}^{\prime}\right), 0\right) \times K_{D}\left(\bar{z}^{\prime \prime}, \beta \mid \bar{z}^{\prime}, 0\right),
\end{aligned}
$$

where the vector $\bar{x}$ denotes the $N$-dimensional $x$ coordinates of the particles, with the notation $\bar{x}^{T}=\left(x_{1}, x_{2}, \ldots, x_{N}\right)$, and similarly for $\bar{y}$ and $\bar{z}$. The propagator for a single oscillator with frequency $\varpi$ in a magnetic field is well known [3] and given by

$$
\begin{aligned}
K_{\omega_{L}}^{(1)}\left(\mathbf{r}^{\prime \prime}, \beta \mid \mathbf{r}^{\prime}, 0\right)= & \sqrt{\frac{\varpi}{2 \pi \sinh \beta \varpi}} \frac{s}{2 \pi \sinh \beta s} \exp \left\{-\frac{\varpi}{2 \sinh \beta \varpi}\left\{\left[\left(z^{\prime}\right)^{2}+\left(z^{\prime \prime}\right)^{2}\right] \cosh \beta \varpi-2 z^{\prime} z^{\prime \prime}\right\}\right\} \\
& \times \exp \left\{-\frac{s}{2} \frac{\left[\left(x^{\prime \prime}\right)^{2}+\left(y^{\prime \prime}\right)^{2}+\left(x^{\prime}\right)^{2}+\left(y^{\prime}\right)^{2}\right] \cosh \beta s-2\left(x^{\prime} x^{\prime \prime}+y^{\prime} y^{\prime \prime}\right) \cosh \beta \omega_{L}}{\sinh \beta s}\right\} \\
& \times \exp \left\{-i\left(\omega_{L}\left(x^{\prime \prime} y^{\prime \prime}-x^{\prime} y^{\prime}\right)-s \frac{\sinh \beta \omega_{L}}{\sinh \beta s}\left(y^{\prime} x^{\prime \prime}-y^{\prime \prime} x^{\prime}\right)\right)\right\},
\end{aligned}
$$

where $\omega_{L}=\omega_{c} / 2$ is the Larmor frequency and the eigenfrequency $s$ is given by

$$
s=\sqrt{\varpi^{2}+\omega_{L}^{2}}
$$

The propagator for $N$ distinguishable interacting oscillators in a magnetic field thus becomes

$$
\begin{aligned}
K_{D}\left(\overline{\mathbf{r}}^{\prime \prime}, \beta \mid \overline{\mathbf{r}}^{\prime}, 0\right)= & \frac{K_{\Omega}\left(\sqrt{N} Z^{\prime \prime}, \beta \mid \sqrt{N} Z^{\prime}, 0\right)}{K_{w}\left(\sqrt{N} Z^{\prime \prime}, \beta \mid \sqrt{N} Z^{\prime}, 0\right)} \frac{K_{\omega_{L}, s_{\text {c.m. }}}\left(\sqrt{N} X^{\prime \prime}, \sqrt{N} Y^{\prime \prime}, \beta \mid \sqrt{N} X^{\prime}, \sqrt{N} Y^{\prime}, 0\right)}{K_{\omega_{L}, s}\left(\sqrt{N} X^{\prime \prime}, \sqrt{N} Y^{\prime \prime}, \beta \mid \sqrt{N} X^{\prime}, \sqrt{N} Y^{\prime}, 0\right)} \\
& \times \prod_{j=1}^{N} K_{\omega_{L}, s}\left(x_{j}^{\prime \prime}, y_{j}^{\prime \prime}, \beta \mid x_{j}^{\prime}, y_{j}^{\prime}, 0\right) K_{w}\left(z_{j}^{\prime \prime}, \beta \mid z_{j}^{\prime}, 0\right),
\end{aligned}
$$


where

$$
s=\sqrt{w^{2}+\omega_{L}^{2}} \quad \text { and } \quad s_{\mathrm{c.m} .}=\sqrt{\Omega^{2}+\omega_{L}^{2}} .
$$

The factor $\sqrt{N}$ in the center-of-mass coordinates in Eq. (10) describes the mass $N$ (in atomic units) of the center. The denominator in Eq. (10) accounts for the fact that the internal degrees of freedom are linearly dependent because of the subsidiary conditions (6). Intuitively this factor is quite natural, because the propagator would be the product of oneparticle propagators [3] if the particles were independent.

Knowing the propagator for distinguishable particles, the symmetrized density matrix $K_{I}$ for identical particles can be obtained through the appropriate symmetric or antisymmetric projection

$$
K_{I}\left(\overline{\mathbf{r}}^{\prime \prime}, \beta \mid \overline{\mathbf{r}}^{\prime}, 0\right)=\frac{1}{N !} \sum_{P} \xi^{p} K_{D}\left(P \overline{\mathbf{r}}^{\prime \prime}, \beta \mid \overline{\mathbf{r}}^{\prime}, 0\right),
$$

where $P$ denotes the permutation matrix, with $\xi=+1$ for bosons and $\xi=-1$ for fermions. Even for this harmonic model, with or without magnetic field, the sum over the permutations has to remain rather formal at the level of the propagator. However, for the partition function
$Z_{I}(\beta, N)=\int d \overline{\mathbf{r}} K_{I}(\overline{\mathbf{r}}, \beta \mid \overline{\mathbf{r}}, 0)=\frac{1}{N !} \sum_{P} \xi^{p} \int d \overline{\mathbf{r}} K_{D}(P \overline{\mathbf{r}}, \beta \mid \overline{\mathbf{r}}, 0)$,

analytical progress can be made with this summation as will be discussed in the next section. First of all, one has to deal with the center-of-mass contribution to the propagator. Afterwards, the summation over all possible permutations will be rewritten as a summation over all possible cycles.

\section{GENERATING FUNCTION OF THE PARTITION FUNCTION}

The center of mass is not independent of the positions of the other particles, which complicates the calculation of the trace of the propagator. To deal with the contribution of the center-of-mass coordinate $\mathbf{R}$ to the propagator, we introduce the delta function $\delta\left(\mathbf{R}-(1 / N) \sum_{j=1}^{N} \mathbf{r}_{j}\right)$ in its Fourier representation as in [3]. This $\delta$ function allows one to formally treat the center-of-mass coordinate as an independent variable. Applying this identity to the partition function, one ends up with

$$
\begin{aligned}
Z_{I}(\beta, N)= & \int d \mathbf{R} \int \frac{d \mathbf{k}}{(2 \pi)^{3}} e^{i \mathbf{k} \cdot \mathbf{R}} \frac{K_{\Omega}(\sqrt{N} Z, \beta \mid \sqrt{N} Z, 0)}{K_{w}(\sqrt{N} Z, \beta \mid \sqrt{N} Z, 0)} \frac{K_{\omega_{L, s} s_{\mathrm{c} . \mathrm{m}}}(\sqrt{N} X, \sqrt{N} Y, \beta \mid \sqrt{N} X, \sqrt{N} Y, 0)}{K_{\omega_{L, s}}(\sqrt{N} X, \sqrt{N} Y, \beta \mid \sqrt{N} X, \sqrt{N} Y, 0)} \\
& \times \int d \overline{\mathbf{r}} \frac{1}{N !} \sum_{P} \xi^{p} \prod_{j=1}^{N} K_{\omega_{L},}\left((P x)_{j},(P y)_{j}, \beta \mid x_{j}, y_{j}, 0\right) K_{w}\left((P z)_{j}, \beta \mid z_{j}, 0\right) e^{-i \vec{k} \cdot \vec{r}_{j} / N} .
\end{aligned}
$$

The problem at hand is the rewriting of the summation over the permutations as a sum over all possible cycles [3]. This cyclic decomposition requires the solution of the path integral for a driven harmonic oscillator in a magnetic field, which is discussed in the Appendix.

A permutation can be decomposed into $M_{\ell}$ cycles of length $\ell$, and the positive integers $M_{\ell}$ and $\ell$ have to satisfy the constraint

$$
\sum \ell M_{\ell}=N
$$

The number $M\left(M_{1}, \ldots, M_{N}\right)$ of permutations with $M_{1}$ cycles of length $1, \ldots, M_{\ell}$ cycles of length $\ell, \ldots$ is given by $M\left(M_{1}, \ldots, M_{N}\right)=N ! /\left[\Pi_{\ell} M_{\ell} ! \ell^{M} /\right]$. Furthermore, a cycle of length $\ell$ will be obtained from $\ell-1$ permutations. Thus, the sign factor $\xi^{p}$ can be rewritten as $\xi^{p}$ $=\Pi_{\ell} \xi^{(\ell-1) M_{\ell}}$. These considerations enable one to rewrite the partition function as

$$
\begin{aligned}
Z_{I}(\beta, N)= & \int d \mathbf{R} \int \frac{d \mathbf{k}}{(2 \pi)^{3}} e^{i \mathbf{k} \cdot \mathbf{R}} \frac{K_{\Omega}(\sqrt{N} Z, \beta \mid \sqrt{N} Z, 0)}{K_{w}(\sqrt{N} Z, \beta \mid \sqrt{N} Z, 0)} \\
& \times \frac{K_{\omega_{L} s_{\text {c.m. }} .}(\sqrt{N} X, \sqrt{N} Y, \beta \mid \sqrt{N} X, \sqrt{N} Y, 0)}{K_{\omega_{L}, s}(\sqrt{N} X, \sqrt{N} Y, \beta \mid \sqrt{N} X, \sqrt{N} Y, 0)} \\
& \times \sum_{M_{1}, \ldots, M_{N}} \prod_{j=1}^{N} \frac{\xi^{(\ell-1) M_{\ell}}}{M_{\ell ! \ell^{M}}}[\mathcal{K} /(\mathbf{k})]^{M /},
\end{aligned}
$$

with

$$
\begin{aligned}
\mathcal{K}_{\ell}(\mathbf{k})= & \int d \mathbf{r}_{\ell+1} \cdots \int d \mathbf{r}_{1} \delta\left(\mathbf{r}_{\ell+1}-\mathbf{r}_{1}\right) \\
& \times \prod_{j=1}^{\ell} K_{\omega_{L},}\left(x_{j+1}, y_{j+1}, \beta \mid x_{j}, y_{j}, 0\right) \\
& \times K_{w}\left(z_{j+1}, \beta \mid z_{j}, 0\right) e^{-i \vec{k} \cdot \vec{r}_{j} / N} .
\end{aligned}
$$

The $\delta$ function explicitly indicates that the trace is taken over a cycle of length $\ell$. It is obvious that $\mathcal{K} /(\mathbf{k})$ factorizes as 


$$
\mathcal{K}_{\ell}(\mathbf{k})=\mathcal{K}_{\ell}\left(k_{x}, k_{y}\right) \mathcal{K}_{\ell}\left(k_{z}\right) .
$$

Taking into account the semigroup property of the propagators $K_{\omega_{L}, s}\left(x_{j+1}, y_{j+1}, \beta \mid x_{j}, y_{j}, 0\right)$ and $K_{w}\left(z_{j+1}, \beta \mid z_{j}, 0\right)$, one immediately recognizes in $\mathcal{K}_{\ell}(\mathbf{k})$ the partition function of a driven harmonic oscillator in a magnetic field, with the driving force

$$
\mathbf{f}(\tau)=i \frac{\mathbf{k}}{N} \sum_{j=0}^{\ell-1} \delta(\tau-j \beta) .
$$

The calculation of the propagator and the partition function for a driven harmonic oscillator in a magnetic field, with the Lagrangian

$$
\begin{aligned}
L_{\mathbf{f}, \omega_{L}}^{(1)}= & \frac{1}{2}\left(\dot{x}^{2}+\dot{y}^{2}\right)-2 \omega_{L} x \dot{y}-\frac{w^{2}}{2}\left(x^{2}+y^{2}\right)+f_{x}(\tau) x \\
& +f_{y}(\tau) y,
\end{aligned}
$$

is illustrated in the Appendix. As mentioned above, the hard core of this approach is the evaluation of the classical action, but a fully stochastic method [24] could as well have been followed. After tedious algebra one eventually finds for the partition function

$$
\begin{aligned}
Z_{I}(\beta, N)= & \int d \mathbf{R} \frac{K_{\Omega}(\sqrt{N} Z, \beta \mid \sqrt{N} Z, 0)}{K_{w}(\sqrt{N} Z, \beta \mid \sqrt{N} Z, 0)} \frac{K_{\omega_{L,} s_{\mathrm{c} . \mathrm{m} .}}(\sqrt{N} X, \sqrt{N} Y, \beta \mid \sqrt{N} X, \sqrt{N} Y, 0)}{K_{\omega_{L,}{ }^{s}}(\sqrt{N} X, \sqrt{N} Y, \beta \mid \sqrt{N} X, \sqrt{N} Y, 0)} \int \frac{d \mathbf{k}}{(2 \pi)^{3}} e^{i \mathbf{k} \cdot \mathbf{R}} \\
& \times \exp \left(-\frac{1}{4 s} \frac{k_{x}^{2}+k_{y}^{2}}{N} \frac{\sinh \beta s}{\cosh \beta s-\cosh \beta \omega_{L}}-\frac{1}{4 w} \frac{k_{z}^{2}}{N} \frac{\sinh \beta w}{\cosh \beta w-1}\right) \\
& \times \sum_{M_{1}, \ldots, M_{N}} \prod_{j=1}^{N} \frac{\xi^{(\ell-1) M_{\ell}}}{M_{\ell} ! \ell^{M} \ell}\left(\frac{1}{8 \sinh \left[\ell \beta\left(s+\omega_{L}\right) / 2\right] \sinh \left[\ell \beta\left(s-\omega_{L}\right) / 2\right] \sinh (\ell \beta w / 2)}\right)^{M_{\ell}} .
\end{aligned}
$$

The remaining integrations over $\mathbf{k}$ and $\mathbf{R}$ are Gaussian and relatively easy to perform, leading to

$$
Z_{I}(\beta, N)=\frac{\sinh \left[\beta\left(s+\omega_{L}\right) / 2\right] \sinh \left[\beta\left(s-\omega_{L}\right) / 2\right] \sinh (\beta w / 2)}{\sinh \left[\beta\left(s_{\text {c.m. }}+\omega_{L}\right) / 2\right] \sinh \left[\beta\left(s_{\text {c.m. }}-\omega_{L}\right) / 2\right] \sinh (\beta \Omega / 2)} Z_{I}(N),
$$

with

$$
\mathbb{Z}_{I}(\beta, N)=\sum_{M_{1}, \ldots, M_{N}} \prod_{j=1}^{N} \frac{\xi^{(\ell-1) M_{\ell}}}{M_{\ell} ! \ell^{M_{\ell}}}\left(\frac{1}{8 \sinh \left[\ell \beta\left(s+\omega_{L}\right) / 2\right] \sinh \left[\ell \beta\left(s-\omega_{L}\right) / 2\right] \sinh (\ell \beta w / 2)}\right)^{M_{\ell}} .
$$

The contribution $\mathbb{Z}_{I}(\beta, N)$ derives from the internal degrees of freedom, treated as independent particles. It contains the full influence of the statistics of the particles, and leads to the true partition function $Z_{I}(\beta, N)$ by multiplication with a simple analytical factor. In practice, the condition (15) complicates the use of the above expression for the partition function for a large number of particles. However, this difficulty can be overcome through the use of the generating function. From the generating function one can then extract the partition function through an inversion of its defining Taylor series.

\section{Generating function and recurrence relation for the partition function}

The generating function technique was used before [3] to obtain the partition function of a set of harmonically interacting identical oscillators in the absence of a magnetic field. In the presence of a magnetic field, a similar construction can be used. Introducing the generating function as

$$
\Xi_{I}(\beta, u)=\sum_{N=0}^{\infty} Z_{I}(N) u^{N}
$$

[with $\mathbb{Z}_{I}(\beta, 0)=1$ by definition], the partition function for the internal degrees of freedom can be obtained from

$$
\mathbb{Z}_{I}(\beta, N)=\left.\frac{1}{N !} \frac{d^{N}}{d u^{N}} \Xi_{I}(u)\right|_{u=0} .
$$

The generating function itself can be obtained with straightforward algebra

$$
\Xi_{I}(\beta, u)=\exp \left(\sum_{\ell=1}^{\infty} \frac{\xi^{\ell-1}}{\ell} \frac{\left(b_{1} b_{2} b\right)^{\ell / 2} u^{\ell}}{\left(1-b^{\ell}\right)\left(1-b_{1}^{\ell}\right)\left(1-b_{2}^{\ell}\right)}\right)
$$

with the notation

$$
b=e^{-\beta w}, \quad b_{1}=e^{-\beta\left(s+\omega_{L}\right)}, \quad b_{2}=e^{-\beta\left(s-\omega_{L}\right)} .
$$

The cyclic summation can be rewritten in terms of the occupation number representation which directly involves the single-particle energy levels: 


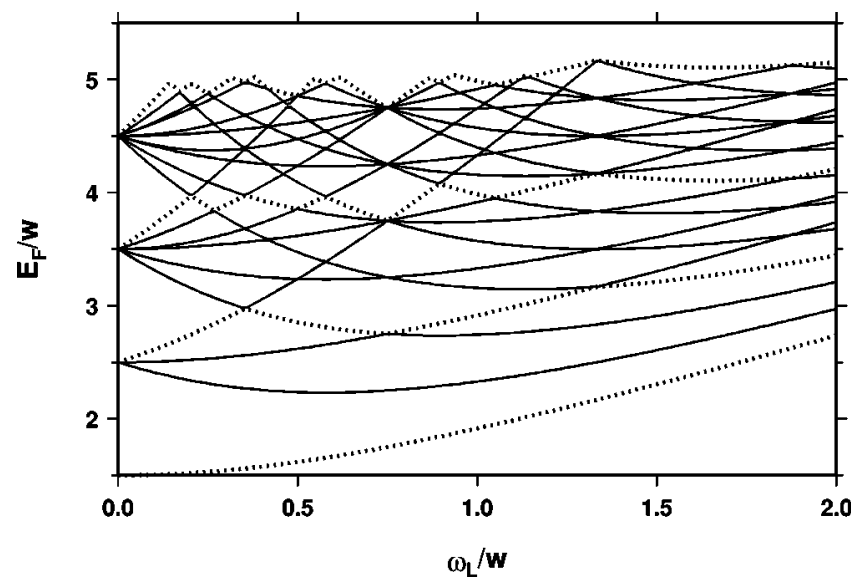

FIG. 1. Lowest single-particle energy levels (in units of $w$ ) as a function of the Larmor frequency. The Fermi energies corresponding to $1,4,10$, and 20 fermions (i.e., for closed shells in the absence of a magnetic field) are emphasized by dashed lines. The results can also be found in Ref. [25].

$$
\Xi_{I}(\beta, u)=\prod_{\nu, \nu_{1}, \nu_{2}=0}^{\infty}\left(1-\xi u b_{1}^{\nu_{1}+1 / 2} b_{2}^{\nu_{2}+1 / 2} b^{\nu+1 / 2}\right)^{-\xi}
$$

By simply applying the chain rule, the expression (25) for the partition function can be written as a recurrence relation

$$
\begin{aligned}
\mathbb{Z}_{I}(\beta, N)= & \frac{1}{N} \sum_{\ell=1}^{N} \xi^{\ell-1} \frac{\left(b_{1} b_{2} b\right)^{\ell / 2}}{\left(1-b_{1}^{\ell}\right)\left(1-b_{2}^{\ell}\right)\left(1-b^{\ell}\right)} \\
& \times \mathbb{Z}_{I}(\beta, N-\ell) .
\end{aligned}
$$

However, if the number of particles increases, this recurrence relation becomes numerically unpractical because of a numerical sign problem for fermions and drastically increasing simulation time for bosons. For the remaining part of this paper, the attention will be focussed on the fermion case.

\section{THERMODYNAMICAL PROPERTIES}

The thermodynamical properties of the fermion model can in essence be determined from the contribution $\mathbb{Z}_{F}(\beta, N)$ of the internal degrees of freedom. As is clear from Eq. (22), the center-of-mass correction only adds a trivial contribution to the free energy. All the effects of the fermion statistics are collected in $Z_{F}(\beta, N)$. We first study the zero-temperature limit, in which special attention will be paid to the twodimensional case in the $x y$ plane, and subsequently the evolution of the free energy and the internal energy as a function of the temperature and of the magnetic field.

\section{A. Zero-temperature limit}

The ground state properties of the fermion model crucially depend on the single-particle energy levels $E_{\nu, \nu_{1}, \nu_{2}}$. These levels and their occupation by fermions have been discussed and plotted earlier, e.g., in Ref. [25]. For easier reference, we plot the 20 lowest levels Fig. 1. To guide the eye, the Fermi energies $E_{F}$ corresponding to the fully occupied levels at $\omega_{L}=0$ are indicated by the dashed line. Note

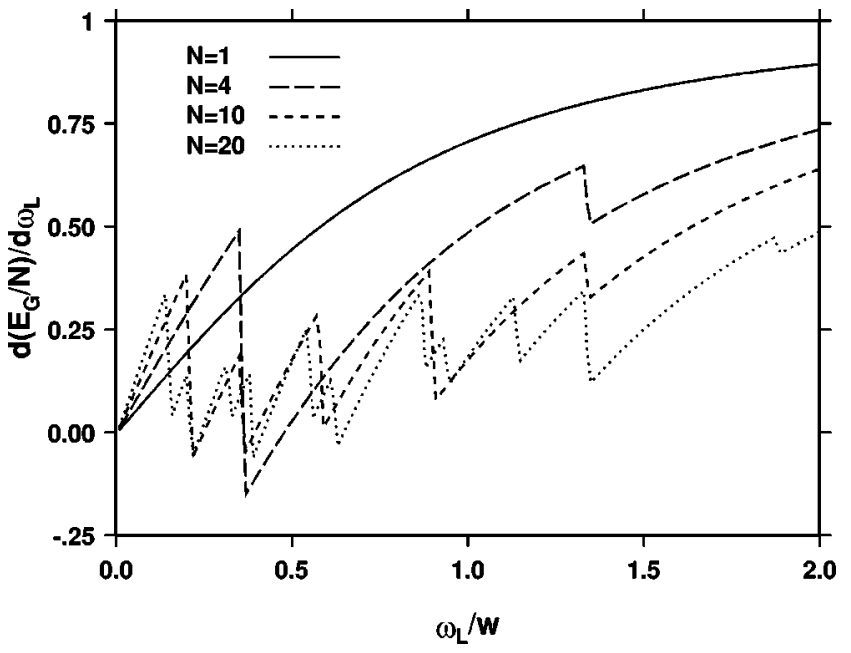

FIG. 2. Scaled magnetic susceptibility $(1 / N)\left(d E_{G} / d \omega_{L}\right)$ as a function of the magnetic field for $1,4,10$, and 20 fermions in the ground state.

that the magnetic field does not substantially influence the magnitude of the Fermi energy, which remains of order $N^{1 / 3}$ for sufficiently large $N$. The magnetic field immediately lifts the degeneracy, but with increasing magnetic field other degeneracies appear and disappear again at particular values of the magnetic field. Although these degeneracies have little effect on the magnitude of the ground state energy $E_{G}$ $=\Sigma_{E<E_{F}} E$, they have a drastic effect on the magnetic susceptibility, which is proportional to $d E_{G} / d \omega_{L}$, as shown in Fig. 2 as a function of the magnetic field. The discontinuities in the magnetic susceptibility occur at those values of the magnetic field where the single-particle energies become degenerate.

\section{B. Free energy and internal energy}

As mentioned above, the sign problem for fermions can be worked around by inverting the defining Taylor series (24) for the generating function. The Fowler-Darwin method [26] provides an accurate and elegant way [27] to realize this inversion:

$$
\mathbb{Z}_{F}(\beta, N)=\frac{1}{2 \pi i} \oint_{C} \frac{\Xi_{F}(\beta, z)}{z^{N+1}} d z
$$

If one considers a circular contour $z=u e^{i \theta}$ with radius $u$, an optimal value of $u$ can be determined by the method of steepest descent:

$$
\frac{d}{d u}\left[\ln \Xi_{F}(\beta, u)-N \ln u\right]=0 \Rightarrow N=u \frac{d}{d u} \ln \Xi_{F}(\beta, u) .
$$

Using Eq. (28), this condition becomes

$$
N=\sum_{\nu, \nu_{1}, \nu_{2}=0}^{\infty} n_{\nu, \nu_{1}, \nu_{2}}
$$

with 


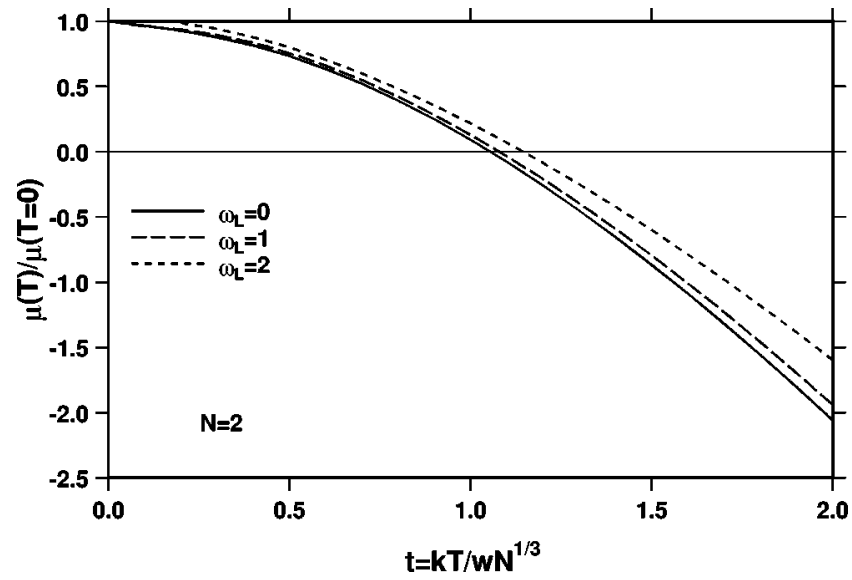

FIG. 3. Scaled chemical potential $\mu(T) / \mu(T=0)$ as a function of the scaled temperature $t=k T / w N^{1 / 3}$ for 2 fermions and for $\omega_{L} / w=0,1$, and 2 .

$$
\begin{gathered}
n_{\nu, \nu_{1}, \nu_{2}}=\frac{1}{1+e^{\beta E_{\nu, \nu_{1}, \nu_{2} u}}} \\
E_{\nu, \nu_{1}, \nu_{2}}=\left(\nu_{1}+\frac{1}{2}\right) s_{1}+\left(\nu_{2}+\frac{1}{2}\right) s_{2}+\left(\nu+\frac{1}{2}\right) w
\end{gathered}
$$

If $u$ were to be interpreted as the fugacity $u=e^{\beta \mu}$ with chemical potential $\mu$, one thus would recover similar results as for the expectation value of the number of particles in the grand canonical ensemble. The result for the chemical potential as a function of temperature is shown in Fig. 3 for various values of the magnetic field and for $N=2$. The chemical potential is plotted in units of the chemical potential at $T$ $=0$ and the temperature in units of $w N^{1 / 3}$, which is the order of magnitude for the Fermi energy. In Figs. 4 and 5 the corresponding results are shown for $N=10$ and $N=100$. For $N \gtrsim 100$ it turns out that $\mu(T) / \mu(T=0)$ as a function of $k T / w N^{1 / 3}$ becomes almost independent of both the number of particles and of the magnetic field.

However, in the present treatment the determination of $u=e^{\beta \mu}$ from Eq. (32) only gives the zero-order contribution to the partition function. A correction by the integration fac-

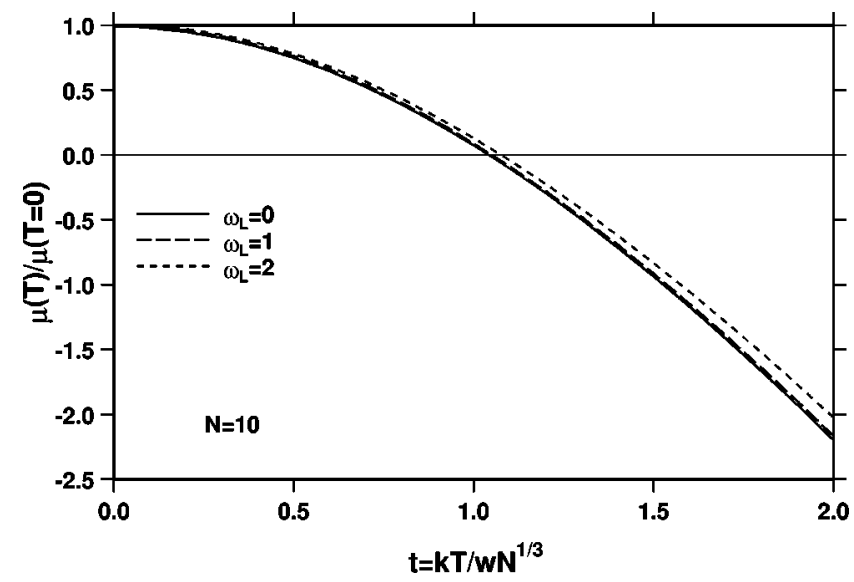

FIG. 4. Same as Fig. 3, but for 10 fermions.

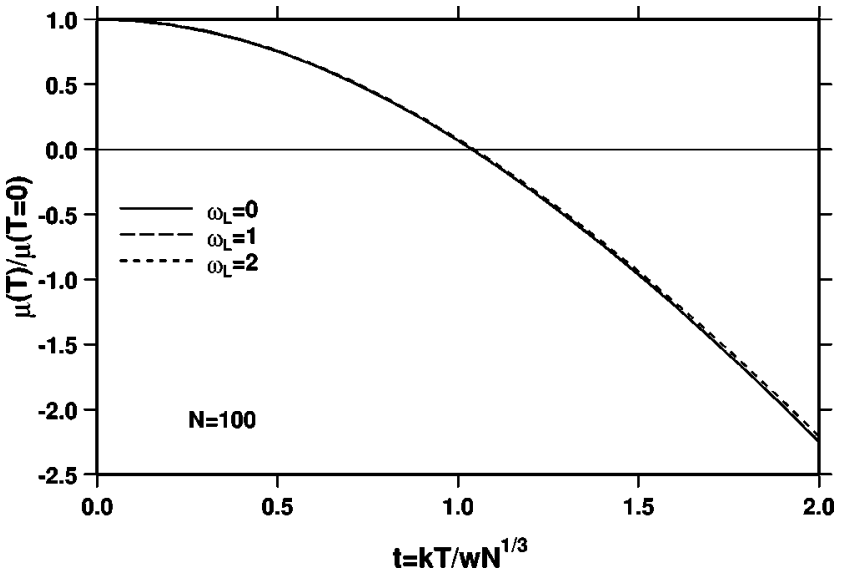

FIG. 5. Same as Fig. 3, but for 100 fermions.

tor in Eq. (30) has to be applied. Using the symmetry of the integrand in Eq. (30), the partition function can be rewritten as

$$
\begin{aligned}
\mathbb{Z}_{F}(\beta, N) & =\frac{\Xi_{F}(\beta, u)}{u^{N}} \frac{1}{2 \pi} \int_{0}^{2 \pi \Xi_{F}\left(\beta, u e^{i \theta}\right)} \frac{\Xi_{F}(\beta, u)}{\Xi^{-i N \theta} d \theta} \\
& =\frac{\Xi_{F}(\beta, u)}{u^{N}} \int_{0}^{\pi} \Psi(\theta) d \theta,
\end{aligned}
$$

with

$$
\Psi(\theta)=\operatorname{Re}\left[\frac{1}{\pi} e^{-i \theta N} \frac{\Xi_{F}\left(\beta, u e^{i \theta}\right)}{\Xi_{F}(\beta, u)}\right] .
$$

The function $\Psi(\theta)$ has to be calculated and integrated numerically. The determination of the free energy

$$
\mathbb{F}_{F}(\beta, N)=-\frac{1}{N} \ln \mathbb{Z}_{F}(\beta, N)=\mathbb{F}_{F}^{(0)}(\beta, N)-\frac{1}{\beta} \ln \int_{0}^{\pi} \Psi(\theta) d \theta
$$

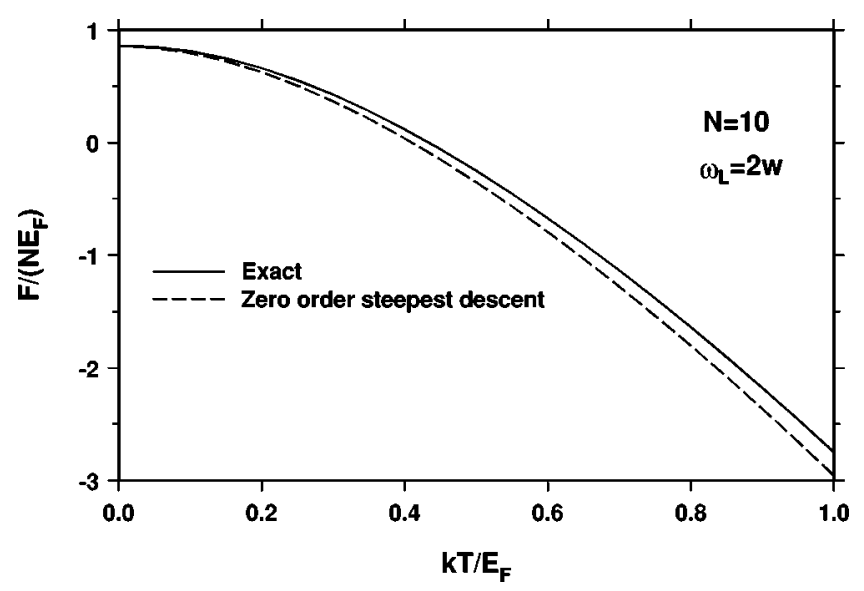

FIG. 6. Scaled free energy per particle $f=\mathbb{F}_{F} / N E_{F}$ as a function of the scaled temperature $k T / E_{F}$ for 10 fermions and with the Larmor frequency $\omega_{L}=2 w$. The zero-order "steepest descent" contribution is indicated by the dashed line. 


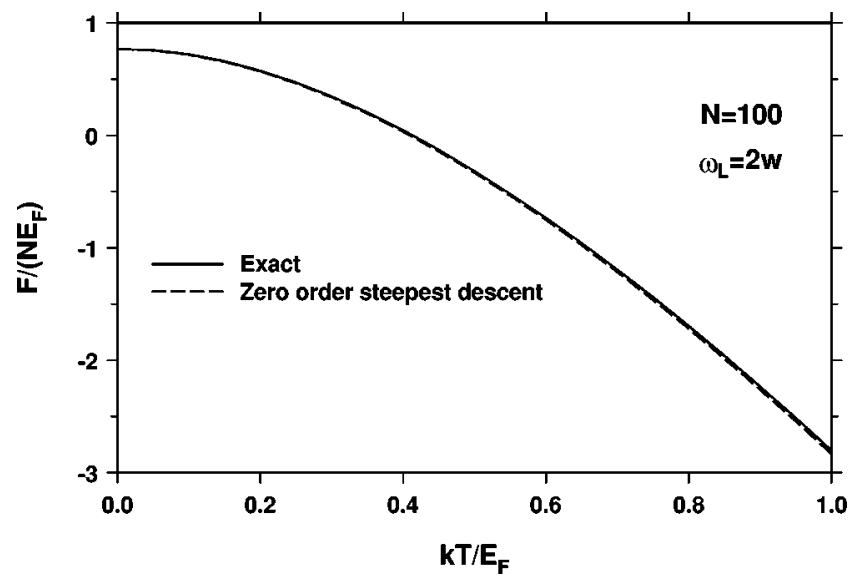

FIG. 7. Same as Fig. 6, but for 100 fermions.

then becomes straightforward, with the zero-order contribution

$$
\mathbb{F}_{F}^{(0)}(\beta, N)=-\frac{1}{\beta} \ln \frac{\Xi_{F}(\beta, u)}{u^{N}}
$$

from the steepest descent approximation. In the absence of a magnetic field, the results are discussed in [6]. For $\omega_{L}=2 w$ the free energy per particle in units of Fermi energy is plotted as a function of $k T / E_{F}$ for 10 fermions in Fig. 6 and for 100 fermions in Fig. 7, and compared to the zero-order steepest descent contribution. Again, for $N \gtrsim 100$ the finite number corrections upon the thermodynamical limit become negligible for all practical purposes. The internal energy $U_{F}$ $=(d / d \beta)\left(\beta \mathbb{F}_{F}\right)$ shows the same universality, as is shown in Fig. 8 where the internal energy per particle in units of the Fermi energy is plotted versus the temperature in units of the Fermi temperature for $\omega_{L}=0, w$, and $2 w$.

\section{CONCLUSION AND DISCUSSION}

Using the path integral approach of symmetrized density matrices for identical particles, the thermodynamical properties were calculated for a three-dimensional model of $N$ harmonically interacting spin-polarized fermions in a parabolic potential well in the presence of a magnetic field. The method used is a generalization of the procedure developed earlier in the absence of a magnetic field. Explicit results were obtained for the ground state energy, the free energy, and the internal energy for a limited number of particles. The model can be described as a number of spin polarized identical particles in a parabolic confinement potential interacting through a special many-body interaction with the consequence that the center of mass is allowed to move independent from the other degrees of freedom. For an analogous model other forms of confinement potentials have been investigated without two-body interaction [29]. With two-body interactions, the model has been studied with the operator formalism in several papers [30,31], with emphasis on the ground state properties.

The statistics with a finite number of particles in the confinement potential and the crossover to density-dependent expressions known from the thermodynamical limit can be studied in this model: as soon as the number of fermions is

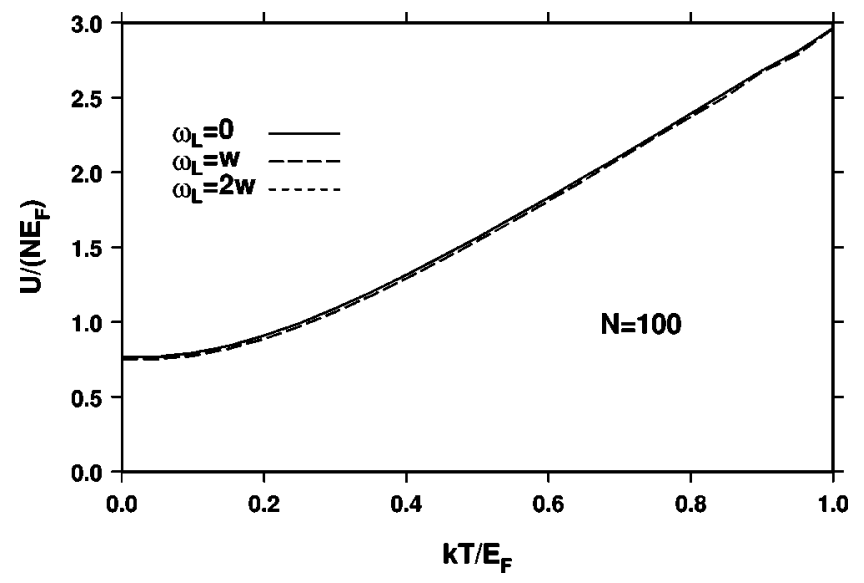

FIG. 8. Scaled internal energy per particle $u=\mathrm{U}_{F} / N E_{F}$ for 100 fermions as a function of the scaled temperature $k T / E_{F}$ for several values of the Larmor frequency $\omega_{L}=0, w$, and $2 w$.

sufficiently large (in the order of $N \gtrsim 100$ ) the results are shown to agree and the finite number corrections become relatively small. The internal energy turns out to obey a scaling law, similar to the scaling from the continuum approximation for the density of states.

\section{ACKNOWLEDGMENTS}

Part of this work was performed in the framework of FWO Projects Nos. 1.5.729.94, 1.5.545.98, G.0287.95, G.0071.98, and WO.073.94N (Wetenschappelijke Onderzoeksgemeenschap over "Laag-dimensionele systemen"), the "Interuniversitaire Attractiepolen-Belgische Staat, Diensten van de Eerste Minister-Wetenschappelijke, and Technische en Culturele aangelegenheden,' and in the framework of the BOF NOI 1997 projects of the Universiteit Antwerpen. One of the authors (F.B.) acknowledges the FWO (Fonds voor Wetenschappelijk OnderzoekVlaanderen) for financial support. S.F. acknowledges the University of Antwerpen (UIA) for a research grant.

\section{APPENDIX: THE PATH INTEGRAL OF THE MODEL FOR DISTINGUISHABLE PARTICLES \\ IN THE PRESENCE OF A MAGNETIC FIELD AND A TIME-DEPENDENT DRIVING FORCE}

The propagator of a two-dimensional harmonic oscillator in the presence of a magnetic field, characterized by the Larmor frequency $\omega_{L}=\omega_{c} / 2$, and under the influence of a timedependent driving force $\mathbf{f}=\left(f_{x}, f_{y}\right)$, provides the basic building blocks for the harmonic model system of identical interacting particles which is the subject of the present paper. Although the calculation of this propagator relies on standard techniques, to the best of our knowledge it is not documented in the literature. Therefore we discuss its derivation here in some detail. The Lagrangian under consideration is given by (in atomic units $\hbar=m=|e|=1$ )

$$
\begin{aligned}
L_{\mathbf{f}, \omega_{L}}^{(1)}= & \frac{1}{2}\left(\dot{x}^{2}+\dot{y}^{2}\right)-2 \omega_{L} x \dot{y}-\frac{w^{2}}{2}\left(x^{2}+y^{2}\right)+f_{x}(\tau) x \\
& +f_{y}(\tau) y .
\end{aligned}
$$


The classical equations of motion in Euclidean time $\tau=i t$ are

$$
\begin{gathered}
-\frac{d^{2} x}{d \tau^{2}}=-2 i \omega_{L} \frac{d y}{d \tau}-w^{2} x+f_{x}, \\
-\frac{d^{2} y}{d \tau^{2}}=2 i \omega_{L} \frac{d x}{d \tau}-w^{2} y+f_{y} .
\end{gathered}
$$

This set of coupled differential equations can be solved and yields

$$
x(\tau)=x_{h}(\tau)+x_{p}(\tau), \quad y(\tau)=y_{h}(\tau)+y_{p}(\tau)
$$

The solutions $x_{h}(\tau)$ and $y_{h}(\tau)$ of the homogeneous equations of motion (without the driving force), which exhaust the boundary conditions $x_{h}(0)=x^{\prime}, x_{h}(\beta)=x^{\prime \prime}, y_{h}(0)$ $=y^{\prime}, y_{h}(\beta)=y^{\prime \prime}$, are found to be

$$
\left(\begin{array}{l}
x_{h}(\tau) \\
y_{h}(\tau)
\end{array}\right)=\frac{\sinh s(\beta-\tau)}{\sinh \beta s}\left(\begin{array}{cc}
\cosh \omega_{L} \tau & i \sinh \omega_{L} \tau \\
-i \sinh \omega_{L} \tau & \cosh \omega_{L} \tau
\end{array}\right)\left(\begin{array}{l}
x^{\prime} \\
y^{\prime}
\end{array}\right)+\frac{\sinh s \tau}{\sinh \beta s}\left(\begin{array}{cc}
\cosh \omega_{L}(\beta-\tau) & -i \sinh \omega_{L}(\beta-\tau) \\
i \sinh \omega_{L}(\beta-\tau) & \cosh \omega_{L}(\beta-\tau)
\end{array}\right)\left(\begin{array}{l}
x^{\prime \prime} \\
y^{\prime \prime}
\end{array}\right)
$$

with

$$
s=\sqrt{\omega_{L}^{2}+w^{2}}
$$

The derivation of the particular solutions $x_{p}(\tau)$ and $y_{p}(\tau)$, with the boundary conditions $x_{p}(0)=x_{p}(\beta)=y_{p}(0)=y_{p}(\beta)=0$, is slightly more involved but eventually results in

$$
\begin{aligned}
\left(\begin{array}{l}
x_{p}(\tau) \\
y_{p}(\tau)
\end{array}\right)= & \frac{1}{s} \frac{\sinh s(\beta-\tau)}{\sinh \beta s} \int_{0}^{\tau}\left(\begin{array}{cc}
\cosh \omega_{L}(\tau-\sigma) & i \sinh \omega_{L}(\tau-\sigma) \\
-i \sinh \omega_{L}(\tau-\sigma) & \cosh \omega_{L}(\tau-\sigma)
\end{array}\right)\left(\begin{array}{l}
f_{x}(\sigma) \\
f_{y}(\sigma)
\end{array}\right) \sinh s \sigma d \sigma \\
& +\frac{1}{s} \frac{\sinh s \tau}{\sinh \beta s} \int_{\tau}^{\beta}\left(\begin{array}{cc}
\cosh \omega_{L}(\tau-\sigma) & i \sinh \omega_{L}(\tau-\sigma) \\
-i \sinh \omega_{L}(\tau-\sigma) & \cosh \omega_{L}(\tau-\sigma)
\end{array}\right)\left(\begin{array}{l}
f_{x}(\sigma) \\
f_{y}(\sigma)
\end{array}\right) \sinh s(\beta-\sigma) d \sigma .
\end{aligned}
$$

Given the classical trajectory with initial position $\left(x^{\prime}, y^{\prime}\right)$ and final position $\left(x^{\prime \prime}, y^{\prime \prime}\right)$ after an imaginary time lapse $\beta$, the corresponding classical action $S_{\mathbf{f}, c l}=\int_{0}^{\beta} L_{\mathbf{f}} d \tau$ can be found by elementary methods. This eventually results in

$$
\begin{aligned}
S_{\mathbf{f}, c l}\left(x^{\prime \prime}, y^{\prime \prime}, \beta \mid x^{\prime}, y^{\prime}, 0\right)=-\frac{s}{\sinh \beta s}\left(\begin{array}{c}
\frac{1}{2}\left[\left(x^{\prime \prime}\right)^{2}+\left(y^{\prime \prime}\right)^{2}+\left(x^{\prime}\right)^{2}+\left(y^{\prime}\right)^{2}\right] \cosh \beta s \\
-\left(x^{\prime} x^{\prime \prime}+y^{\prime} y^{\prime \prime}\right) \cosh \beta \omega_{L}+i\left(x^{\prime} y^{\prime \prime}-x^{\prime \prime} y^{\prime}\right) \sinh \beta \omega_{L}
\end{array}\right)-i \omega_{L}\left(x^{\prime \prime} y^{\prime \prime}-x^{\prime} y^{\prime}\right) \\
+x^{\prime} \int_{0}^{\beta}\left[f_{x}(\tau) \cosh \omega_{L} \tau-i f_{y}(\tau) \sinh \omega_{L} \tau\right] \sinh s(\beta-\tau) d \tau \\
+\frac{1}{\sinh \beta s}\left(\begin{array}{l}
x^{\prime \prime} \int_{0}^{\beta}\left[f_{x}(\tau) \cosh \omega_{L}(\beta-\tau)+i f_{y}(\tau) \sinh \omega_{L}(\beta-\tau)\right] \sinh s \tau d \tau \\
+y^{\prime} \int_{0}^{\beta}\left[f_{y}(\tau) \cosh \omega_{L} \tau+i f_{x}(\tau) \sinh \omega_{L} \tau\right] \sinh s(\beta-\tau) d \tau \\
+y^{\prime \prime} \int_{0}^{\beta}\left[f_{y}(\tau) \cosh \omega_{L}(\beta-\tau)-i f_{x}(\tau) \sinh \omega_{L}(\beta-\tau)\right] \sinh s \tau d \tau
\end{array}\right) \\
+\frac{1}{s \sinh \beta s} \int_{0}^{\beta} \int_{0}^{\tau}\left(\begin{array}{l}
{\left[f_{x}(\tau) f_{x}(\sigma)+f_{y}(\tau) f_{y}(\sigma)\right] \cosh \omega_{L}(\tau-\sigma)} \\
\left.+i(\tau) f_{y}(\sigma)+f_{y}(\tau) f_{x}(\sigma)\right] \sinh \omega_{L}(\tau-\sigma)
\end{array}\right) \sinh \sigma s \sinh s(\beta-\tau) d \sigma d \tau
\end{aligned}
$$

Since the Lagrangian is quadratic in the coordinates and the velocities, the quantum mechanical propagator is determined by the classical action, apart from a trivial normalization factor. The latter can be determined by elementary methods. The calculation presents no difficulties and results in

$$
K_{\mathbf{f}}\left(x^{\prime \prime}, y^{\prime \prime}, \beta \mid x^{\prime}, y^{\prime}, 0\right)=\frac{s}{2 \pi \sinh \beta s} \exp \left[S_{\mathbf{f}, c l}\left(x^{\prime \prime}, y^{\prime \prime}, \beta \mid x^{\prime}, y^{\prime}, 0\right)\right]
$$

If one takes the limit $\omega_{L} \rightarrow 0$, the correct result [1] is recovered.

For the treatment of the cyclic summations for identical particles, it is essential to know the trace of this propagator 


$$
Z_{\mathbf{f}}=\iint K_{\mathbf{f}}(x, y, \beta \mid x, y, 0) d x d y .
$$

The calculation of this quantity is straightforward and after some algebra one obtains

$$
Z_{\mathbf{f}}=\frac{1}{2\left(\cosh \beta s-\cosh \beta \omega_{L}\right)} \exp \left[\frac{\Phi(\beta)}{4 s\left(\cosh \beta s-\cosh \beta \omega_{L}\right)}\right],
$$

with

$$
\begin{aligned}
\Phi(\beta)= & \int_{0}^{\beta} \int_{0}^{\beta}\left[f_{x}(\tau) f_{x}(\sigma)+f_{y}(\tau) f_{y}(\sigma)\right]\left(\begin{array}{c}
\cosh \omega_{L}(\tau-\sigma) \sinh s(\beta-|\tau-\sigma|) \\
+\cosh \omega_{L}(\beta-|\tau-\sigma|) \sinh s(\tau-\sigma)
\end{array}\right) d \sigma d \tau \\
& +i \int_{0}^{\beta} \int_{0}^{\beta}\left[f_{x}(\tau) f_{y}(\sigma)+f_{y}(\tau) f_{x}(\sigma)\right]\left(\begin{array}{c}
\sinh \omega_{L}(\tau-\sigma) \sinh s(\beta-|\sigma-\tau|) \\
-\sinh \omega_{L}(\beta-|\tau-\sigma|) \sinh s(\tau-\sigma)
\end{array}\right) d \sigma d \tau .
\end{aligned}
$$

Again, if one takes the limit of a vanishing magnetic field, one finds the correct result [28].

[1] R. P. Feynman and A. R. Hibbs, Quantum Mechanics and Path Integrals (McGraw-Hill, New York, 1965).

[2] R. P. Feynman, Statistical Mechanics, a Set of Lectures (Benjamin, Reading, MA, 1972).

[3] F. Brosens, J. T. Devreese, and L. F. Lemmens, Phys. Rev. E 55, 227 (1997).

[4] F. Brosens, J. T. Devreese, and L. F. Lemmens, Phys. Rev. E 55, 6795 (1997).

[5] F. Brosens, J. T. Devreese, and L. F. Lemmens, Phys. Rev. E 57, 3871 (1998).

[6] F. Brosens, J. T. Devreese, and L. F. Lemmens, Phys. Rev. E 58, 1634 (1998).

[7] M. H. Anderson, J. R. Ensher, M. R. Matthews, C. E. Wieman, and E. A. Cornell, Science 269, 198 (1995).

[8] K. B. Davis, M. O. Mewes, M. R. Andrews, N. J. van Druten, D. S. Durfee, D. M. Kurn, and W. Ketterle, Phys. Rev. Lett. 75, 3969 (1995).

[9] C. C. Bradlet, C. A. Sackett, J. J. Tollett, and R. G. Hulet, Phys. Rev. Lett. 75, 1687 (1995).

[10] S. Grossman and M. Holthaus, Z. Naturforsch., A: Phys. Sci. 50, 323 (1995); 50, 921 (1995).

[11] S. Grossman and M. Holthaus, Phys. Lett. A 208, 188 (1995).

[12] W. Ketterle and N. J. van Druten, Phys. Rev. A 54, 656 (1996).

[13] K. Kirsten and D. J. Toms, Phys. Rev. A 54, 4188 (1996).

[14] H. Haugerud, T. Haugset, and F. Ravndal, Phys. Lett. A 225, 18 (1997).

[15] L. Cohen and C. Lee, J. Math. Phys. 26, 3105 (1985).

[16] W. Krauth, Phys. Rev. Lett. 77, 3695 (1996).
[17] A. Minguzzi, S. Conti, and M. P. Tosi, J. Phys.: Condens. Matter 9, L33 (1997).

[18] F. Brosens, J. T. Devreese, and L. F. Lemmens, Solid State Commun. 100, 123 (1996).

[19] F. Brosens, J. T. Devreese, and L. F. Lemmens, Phys. Rev. A 55, 2453 (1997).

[20] J. Tempere, F. Brosens, L. F. Lemmens, and J. T. Devreese, Solid State Commun. 107, 51 (1998).

[21] L. F. Lemmens, F. Brosens, and J. T. Devreese, Phys. Rev. E 53, 4467 (1996).

[22] L. F. Lemmens, F. Brosens, and J. T. Devreese, Phys. Lett. A 189, 437 (1994).

[23] F. Brosens, J. T. Devreese, and L. F. Lemmens, Solid State Commun. 96, 137 (1995).

[24] B. Simon, Functional Integration and Quantum Physics (Academic Press, New York, 1979).

[25] L. P. Kouwenhove, T. H. Oosterkamp, S. Tarucha, D. G. Austing, and T. Honda, Physica B 249-251, 191 (1998).

[26] R. Kubo, Statistical Mechanics (North-Holland, Amsterdam, 1965).

[27] L. F. Lemmens, F. Brosens, and J. T. Devreese, Solid State Commun. (to be published).

[28] R. P. Feynman, Phys. Rev. 84, 108 (1951).

[29] P. N. Vorontsov-Velyaminov, S. D. Ivanov, and R. I. Gorbunov, Phys. Rev. E 59, 168 (1999).

[30] N. F. Johnson and M. C. Payne, Phys. Rev. Lett. 67, 1157 (1991); Phys. Rev. B 45, 3819 (1992).

[31] A. Angelucci and A. Tagliacozzo, Phys. Rev. B 56, 7088 (1997). 